\title{
La competencia comunicativa intercultural (CCI) en los contextos de enseñanza del inglés como lengua extranjera*
}

\author{
The Intercultural Communicative Competence (ICC) in the Contexts of Teaching English as a Foreign Language \\ A competência comunicativa intercultural (CCI) nos contextos de ensino do inglês como língua estrangeira
}

Carlos Rico Troncoso ${ }^{\text {a }}$

Pontificia Universidad Javeriana, Colombia

crico@javeriana.edu.co

ORCID: http://orcid.org/0000-0002-4399-3394
DOI: https://doi.org/10.11144/Javeriana.syp37-72.ccic

Redalyc: http://www.redalyc.org/articulo.oa?id=86057225006

Fecha de recepción: 22 Mayo 2018

Fecha de aprobación: 02 Junio 2018

\section{Resumen:}

Este artículo tiene como objetivo presentar una reflexión sobre la forma en cómo se ha entendido la Competencia Comunicativa Intercultural (CCI) en los procesos de enseñanza de lengua extranjera. Para lograr dicho cometido, el texto rastrea en principio lo que ha sido la enseñanza de la lengua extranjera en el contexto de ELT [English Language Teaching]. También se hace una reflexión de lo que implicaría llevar la CCI a un escenario de enseñanza de lengua extranjera. Finalmente, se explica cómo se podría entender la CCI en la enseñanza de lengua extranjera a la luz de tres conceptos que presuponen su definición: la comunicación, la lengua y la cultura.

Palabras clave: Lengua, Comunicación, Cultura, ELT, CCI.

\section{Abstract:}

This article aims to provide a reflection on the way how the Intercultural Communicative Competence (ICC) has been understood in the processes of teaching a foreign language. To attain this goal, this text traces primarily what teaching a foreign language has been so far in the context of ELT [English Language Teaching]. This article then provides another reflection on what is implied when trying to bring the ICC into a foreign language teaching scenario. Finally, it provides an explanation on how to understand the ICC in the teaching of a foreign language through the lens of three concepts that are assumed in its definition: communication, language and culture.

Keywords: language, communication, culture, ELT, ICC.

\section{Resumo:}

Este artigo objetiva apresentar uma reflexão sobre a forma como tem se entendido a Competência Comunicativa Intercultural (CCI) nos processos de ensino de língua estrangeira. Para conseguir tal cometido, o texto rastreja em princípio o que tem sido o ensino da língua estrangeira no contexto de ELT [English Language Teaching]. Mesmo faz-se uma reflexão do que implicaria levar a CCI para um cenário de ensino de língua estrangeira. Por fim, explica como poderia se entender a CCI no ensino de língua estrangeira à luz de três conceitos que pressupõem sua definição: a comunicação, a língua e a cultura.

Palavras-chave: Língua, Comunicação, Cultura, ELT, CCI.

Para responder la pregunta sobre qué significa la enseñanza de lenguas, es posible disertar en numerosas páginas su definición y llegar siempre a la misma conclusión: la enseñanza de lenguas puede ser definida como "todas aquellas actividades que se proponen para lograr el aprendizaje de lenguas" (Stern, 1983, p.21). La definición en sí misma, presupone la relación indisoluble de la enseñanza con el aprendizaje. En teoría, los vínculos entre ambos son incuestionables, sin embargo, lo que se ha visto a través de la historia de la enseñanza de lenguas es que, durante muchos años, se han separado estos dos procesos. Por este motivo un amplio número de métodos y enfoques fueron creados sobre la base de la enseñanza. Es así como la enseñanza

Notas de autor :

a Autor de correspondencia. Correo electrónico: crico@javeriana.edu.co 
de lenguas ha sido ampliamente dominada por teorías basadas en cómo aprenden las personas su lengua materna y cómo los docentes usaban al 'hablante nativo' como modelo para que los aprendientes emplearan dicha lengua. Así las cosas, la proficiencia de lenguas se definía sobre la base de cómo los aprendientes usaban sus habilidades para responder a aspectos puramente gramaticales y qué tanto se asemejaban a los hablantes nativos de la lengua.

Durante las últimas dos décadas, esta tendencia ha cambiado drásticamente dado que el énfasis se ha puesto en los procesos de aprendizaje. La situación radica en que muchas teorías han surgido, pero pocas proveen conocimiento sobre cómo aplicarlas en campos específicos - pensemos en los salones de clase, por ejemplo-. Lo más probable es que ésta sea la razón por la que algunos docentes de lenguas continúan haciendo lo mismo durante años y solo acuñan algunos nombres en nuestras metodologías para sentir que están un poco más 'actualizados'. Ahora, para entender un poco más cómo ha evolucionado la enseñanza de lenguas en el último siglo, y cuál es el énfasis dado en algunas décadas, Stern (1983) presenta cuatro períodos detallando en cada uno de ellos algunas características principales. Con el fin de tener una imagen más amplia de la enseñanza de lenguas, se ha expandido la siguiente tabla (Tabla 1) con un periodo más para cubrir las últimas décadas y así poder resumir las características más importantes por cada periodo. 
TABLA 1

Cambio e innovación en la enseñanza de lenguas

\begin{tabular}{|c|c|c|}
\hline PERIODO & DÉCADA & CARACTERÍSTICAS PRINCIPALES \\
\hline I & $1880-1920$ & $\begin{array}{l}\text { Método directo. La importancia de la fonética (creación de la } \\
\text { Asociación Fonética Intemacional) }\end{array}$ \\
\hline II & $1920-1940$ & $\begin{array}{l}\text { La tragedia de la Primera Guerra Mundial dio lugar a la búsqueda de } \\
\text { una mejor comunicación intemacional. } \\
\text { Surgimiento del enfoque en la lectura propuesto por Incoporated } \\
\text { Association of Assistant Masters en escuelas secundarias en el Reino } \\
\text { Unido. El énfasis de la enseñanza de lenguas estaba en la selección de } \\
\text { vocabulario y en la evaluación. }\end{array}$ \\
\hline III & $\begin{array}{l}1940-1950 \\
1950-1960 \\
1960-1970\end{array}$ & $\begin{array}{l}\text { Surgimiento del enfoque lingüístico en la enseñanza de lenguas. } \\
\text { Los programas de las Fuerzas Amadas demuestran que no es } \\
\text { necesario que la enseñanza de lenguas se realice en cursos } \\
\text { convencionales. Además, se comprueba que la lengua podia } \\
\text { enseñarse a públicos más amplios y, a dicionalmente, se demuestran } \\
\text { las posibles ventajas de una enseñanza intensiva y de un énfasis en la } \\
\text { lengua oral. } \\
\text { Los métodos audio-oral(EU) y audiovisual(Francia/Reino Unido). } \\
\text { Lenguas extranjeras enlas escuelas primarias (Foreign Languages in } \\
\text { the Elementary Schools, FLES, por sus siglas en inglés) } \\
\text { Laboratorios de lengua (y el uso de tecnología como: grabadoras, } \\
\text { radio, televisión, proyectores, instrucción asistida por computa dor) } \\
\text { El surgimiento de la psicolingüística } \\
\text { Teoría del hábito audio-oral vs. Aprendizaje del código cognitivo } \\
\text { (Carroll 1966) } \\
\text { El impacto de la teoria de Chomsky. Sociolingüistica } \\
\text { Investigación delmétodo y el análisis del método (uso del análisis } \\
\text { contrastivo) }\end{array}$ \\
\hline IV & $1970-1980$ & $\begin{array}{l}\text {-Énfasis en el currículo: Actos de habla, análisis de necesidades, } \\
\text { análisis del discurso, lengua para propósitos específicos, inmersión, } \\
\text { niveles de proficiencia. } \\
\text {-Énfasis en la relación humana: Individualización (Autonomía del } \\
\text { aprendiente) Técnicas humanísticas. } \\
\text {-Enfasis en la investigación en el aprendizaje de lenguas: lengua } \\
\text { matemaly segunda lengua, a dquisición/aprendizaje en niñosy } \\
\text { adultos, análisis de errores y estudios de interlenguaje. } \\
\text {-Nuevos métodos: Via silenciosa, Community Language Leaming, } \\
\text { Sugestopedia. }\end{array}$ \\
\hline $\mathbf{v}$ & $1980-2015$ & $\begin{array}{l}\text {-Nuevas tendencias en el Enfoque Comunicativo. Énfasis principal en } \\
\text { las interacciones (interacciones socioculturales) } \\
\text {-El uso de la tecnología y el aprendizaje de autoacceso. } \\
\text {-Enseñanza de competencias (comunicativa e intercultural) } \\
\text {-Enfoque centrado en el aprendizaje y teorias del aprendizaje situa do. } \\
\text {-Perspectivas críticas: Lingüística aplicada crítica (relaciones entre la } \\
\text { lengua, el poder y la identidad-imperialismo lingüístico y el } \\
\text { fenómeno de la estandarización) }\end{array}$ \\
\hline
\end{tabular}

Fuente: Stern (1983). Actualizada por el investigador 
En términos generales, lo que esta tabla muestra es el hecho de que la enseñanza de lenguas ha sido dominada por tres escuelas de pensamiento: Estructural, Funcional e Interaccional. Desde 1880 hasta 1970 la perspectiva predominante fue la estructural. Desde los años 70 hasta el comienzo de los años 90 la perspectiva funcional prevaleció; y desde los años 90 hasta el nuevo siglo la perspectiva interaccional predomina. Ahora miremos en detalle cómo estas perspectivas han definido la enseñanza de lenguas.

La perspectiva Estructural es la más tradicional de las tres. Ésta concibe la lengua como un sistema compuesto por subsistemas que pueden ser estudiados de manera separada (Rico, 2005). Para esta perspectiva, el nivel de proficiencia se determina de acuerdo con la habilidad de los estudiantes para reconocer y dominar los elementos del sistema, lo cual generalmente se define en términos de unidades fonológicas — fonemas-, unidades gramaticales — cláusulas, frases, oraciones_, operaciones gramaticales — agregar, cambiar, juntar o transformar los elementos - y las unidades lexicales — combinación de palabras - (Richards, 1986).

La segunda perspectiva es la funcional. De acuerdo con Richards (1986), la lengua es vista como un vehículo para la expresión de significados funcionales.

Esta perspectiva se enfoca en las dimensiones semántica y comunicativa por encima de las características gramaticales de la lengua, y lleva a la especificación y organización del contenido de la enseñanza de lenguas mediante el uso de categorías de significado y función más que el uso de elementos de estructura y gramática (Richards, 1986, p.17).

Los estudiantes aprenden nociones, temas y conceptos que con el tiempo, emplearán para comunicarse. Bajo esta tendencia se encuentra el Enfoque Comunicativo - Communicative Language Teaching, CLT, su sigla en inglés-, el cual primordialmente se interesa por hacer que los usuarios de la lengua expresen significados en situaciones comunicativas específicas. Una de las debilidades de este método, en la enseñanza de lenguas, es su interpretación de la competencia comunicativa. Esta competencia fue comprendida como "la habilidad para emplear el sistema lingüístico efectiva y apropiadamente" (Richards, 1986, p.67). Para el enfoque comunicativo el significado no es relacional sino, lingüístico. Desde esta mirada, los estudiantes reciben oraciones que tienen que emplear en situaciones comunicativas. Muchas veces estos enunciados no están contextualizados - social o culturalmente-. En este sentido, el contexto es simplemente lingüístico y predecible.

Ahora bien, la tercera perspectiva es la interaccional. Aquí la lengua es vista como un vehículo para llevar a cabo relaciones interpersonales e intercambios sociales entre individuos. Adicionalmente, la lengua también es vista como una herramienta para la creación y el mantenimiento de las relaciones sociales. Desde esta perspectiva, las teorías interaccionales se concentran en los patrones de acciones comunicativas, actos de negociación e interacción que hacen parte de los intercambios conversacionales. Es claro que desde esta mirada, "el contenido de la enseñanza de lenguas se especifica y organiza mediante patrones de intercambios e interacción" (Richards, 1986, p. 17).

En resumen, los paradigmas estructural y funcional, los cuales se pueden llamar como "escuelas clásicas de pensamiento", impiden pensar en la competencia intercultural porque se basan simplemente en el estudio de aspectos formales de la lengua. Del mismo modo, estas perspectivas no relacionan la lengua con otros aspectos importantes vistos en cualquier interacción, tales como los hablantes involucrados, sus intenciones comunicativas, la(s) cultura(s) en contacto presentes en los intercambios y los contextos de interacción. Adicionalmente, estas perspectivas siguen una metodología predecible en donde los estudiantes reciben un input - generalmente lingüístico-, lo practican y luego lo usan en una situación similar. Indudablemente esta posición teórica no invita a los estudiantes a reflexionar sobre el significado de la lengua en términos de sus relaciones dentro del contexto en el que se emplea. Finalmente, como Kramsch (1993) lo afirma, desde estas dos perspectivas no existe una relación entre las formas lingüísticas y las estructuras sociales; es por esta razón que la lengua no es vista como una práctica social. 
En cuanto al hecho de que actualmente, la lengua es vista dentro de la dimensión social y tiene la función intercultural, Buttjes y Byram (1991) resaltan que el 'gran negocio' de la enseñanza de lenguas ha sido menos sensible hacia los problemas interculturales y el contacto de lenguas en situaciones sociales particulares. Por ejemplo, el problema con el inglés como una 'lengua franca' global, consiste en que se le ha dado un valor político y económico como lengua - del gran imperio- que permite los encuentros inter- culturales -lengua puente- - Sería interesante ver cómo los Estados, en su definición de política lingüística, han contribuido al proceso nuevamente de colonización a través de la imposición de una lengua 'universal'. Desde esta mirada, se privilegia un aprendizaje de la lengua extranjera mucho más instrumental que con propósitos integradores - por eso algunos académicos han decidido denominar esta enseñanza con la sigla TENOR o Teaching English for No Obvious Reason-.

\section{La nueva tendencia en la enseñanza de lenguas}

Como se ha mencionado, el objetivo de la enseñanza de una lengua extranjera actualmente no consiste en estudiar la lengua sólo desde la perspectiva formal, sin embargo, también se debe entender que los seres humanos, tienen la capacidad de hacer cosas con la lengua. Sin duda alguna, en la nueva tendencia, uno de los principios más importantes en la enseñanza de lenguas es no realizar ningún tipo de juicio de valor sobre las mismas. Rico (2010) menciona que es muy común escuchar personas que dicen que 'cierta lengua suena mejor que otra' o 'cierto acento es mejor que otro'. Probablemente, estas nociones son heredadas de las escuelas clásicas de pensamiento, en donde las lenguas se clasificaban y se calificaban como 'mejores' o 'peores' por sus usuarios.

El planteamiento anterior, refleja una visión que ha sido superada desde el punto de vista de los estudios del lenguaje sobre las variaciones lingüísticas. Actualmente, el lingüista reconoce y acepta, sin ningún juicio de valor, la existencia de las variedades lingüísticas tales como los dialectos y sociolectos, entre otros conceptos. Tal vez la riqueza en el estudio de cualquier lengua, se concibe más desde las formas como las personas las usan y no cómo deberían usarlas.

En el pasado, la selección de un estándar o norma en la enseñanza de lenguas extranjeras, tendía a ser un imperativo categórico. Esta selección estaba basada en una incuestionable tradición y atribuía prejuicios a favor y en contra de distintas variedades. Como Stern (1983) subraya, en muchos países europeos no es poco común para los docentes de inglés como segunda lengua el hecho de insistir en la enseñanza de lo que ellos, ingenuamente, llaman 'inglés de Oxford' o el 'el inglés de la reina', y rechazan el inglés estadounidense al etiquetarlo como 'menos puro'. Evidentemente, se puede observar que muchas opiniones y nociones sobre la enseñanza de lenguas, están derivadas de los dos paradigmas que enmarcan la idea de que las lenguas pueden ser enseñadas como un sistema formal o estructural y funcional. Stern (1983) ha resumido algunas características las cuales establecen diferencias entre estos dos paradigmas. (Tabla 2) 
TABLA 2

Paradigmas sobre la enseñanza de lenguas

\begin{tabular}{|c|c|}
\hline $\begin{array}{c}\text { Categorias lingüisticas } \\
\text { (Perspectiva estructural y funcional) }\end{array}$ & $\begin{array}{c}\text { Categorias comunicativas } \\
\text { (Perspectiva interaccional) }\end{array}$ \\
\hline exactitud & adecuación \\
\hline Uso (norma)-use & Uso real-usage \\
\hline significado & sentido \\
\hline oración & actuación \\
\hline proposición & Acto ilocutivo \\
\hline cohesión & coherencia \\
\hline Habilidades lingüisticas & Habilidades comunicativas \\
\hline Rasgos lingüisticos & Rasgos socio-culturales \\
\hline
\end{tabular}

Fuente: Stern, 1983, p. 179.

Es posible ver en esta lista de palabras que las categorías comunicativas, apuntan a una perspectiva más holística sobre la lengua y sugiere un análisis de la misma, desde una perspectiva social y cultural. En la práctica, la integración de la lengua con su contexto sociocultural no es un asunto sencillo. Hasta hace poco, el significado social de las formas lingüísticas o de las implicaciones lingüísticas de los hechos sociales no era tenido en cuenta en las didácticas para la enseñanza de las lenguas, ni tampoco hacía parte de los programas de formación de los docentes (Stern, 1983; Rico 2010). Solo hasta en las últimas décadas, han sido reconocidas las contribuciones de las ciencias sociales para una comprensión más amplia de la enseñanza de lenguas.

Stern (1983) afirma que el mayor problema que se enfrenta en la enseñanza de lenguas, es la falta de investigación sociológica y antropológica en las comunidades de habla. Desde el punto de vista de esta investigación, esta tendencia no se ha privilegiado a la hora de definir una política lingüística. Al contrario, lo que se ha hecho desde la promulgación de estas políticas, es tratar de homogenizar y estandarizar procesos que desde su naturaleza son diferentes y variables.

Finalmente, cabe anotar que 'la nueva tendencia' y su relación con las Ciencias Sociales, concibe el rol de la lengua como una práctica social y sugiere nuevas formas de observar la enseñanza de lenguas y la cultura. Es en este sentido que Kramsch (1993b) estableció cuatro necesidades que deberían considerarse en los procesos de enseñanza de una lengua extranjera a saber:

(1) Necesidad de establecer una "esfera de la interculturalidad": Entender una cultura extranjera requiere poner esa cultura en relación con la propia. Cualquier tipo de interacción social, siempre es relacional. Por ejemplo, un enfoque intercultural de la enseñanza de una cultura es radicalmente diferente de la transferencia de información entre culturas. Lo anterior incluye una reflexión tanto de la cultura objetivo como de la propia cultura. 
(2) Necesidad de enseñar la cultura como un proceso intercultural: Si el significado se produce de las interacciones sociales, entonces no tiene ningún sentido intentar enseñar fenómenos fijos y normativos del uso de la lengua. Por el contrario, deberíamos reemplazar la presentación/prescripción de hechos culturales por la enseñanza de un proceso que se aplique para comprender lo 'foráneo' o lo diferente'. Lo anterior implica que los docentes deberían promover que la comunicación, que debería estar en primer plano, se dé manera intercultural o transcultural.

(3) Necesidad de trascender en la visión de la cultura como un proceso diferencial: No podemos ver la cultura únicamente en términos de características nacionales. Por ejemplo, cuando decimos 'los franceses hacen esto' o 'los españoles hacen aquello' podemos fácilmente encontrar dificultades para responder la pregunta ‘ ¿qué significa ser francés o español?’ teniendo en cuenta que estas sociedades son cada vez más multiétnicas y multiculturales. Esto no significa que las cualidades nacionales no sean importantes, sino que estas no pueden ser extraídas sin una profunda especificación de otros factores culturales como la edad, el género, el origen regional, el origen étnico, la clase social y la condición política. Es así como las cualidades nacionales solo son unos de los muchos aspectos de la 'cultura' de una persona.

(4) Necesidad de cruzar los limites disciplinarios: Tenemos que reconocer el vínculo entre la enseñanza de una cultura o de una civilisation (en francés), con disciplinas académicas como la antropología, la sociología e incluso la semiología. Es evidente que se debe invitar a los docentes a ampliar sus concepciones a través de reflexiones motivadas por estudios sociales, etnográficos y sociolingüísticos entre otros. (pp. 205-206)

La mayoría de las ideas presentadas aquí - en la 'nueva tendencia de la enseñanza de lenguas' - han sido contempladas en el Enfoque Comunicativo, el cual ha causado una gran revolución en la pedagogía de las lenguas. Desde la perspectiva de este estudio, el mayor problema en el ámbito académico han sido las malinterpretaciones en cuanto a sus principios. Por ejemplo, como fue mencionado anteriormente, la enseñanza comunicativa de la lengua CLT, en sus inicios malinterpretó la competencia comunicativa, en tanto que no comprendió adecuadamente el concepto de 'contexto' - el cual fue reducido únicamente a la perspectiva lingüística一, y no consideró la variabilidad de la lengua. Para lograr una mejor comprensión, es necesario definir qué es el Enfoque Comunicativo y cómo éste se relaciona con la Competencia Comunicativa Intercultural [CCI], que corresponde al segundo punto de la discusión planteada.

\section{El Enfoque Comunicativo para la Enseñanza de Lenguas}

Cuando se escucha sobre el Enfoque Comunicativo, fácilmente vienen a la mente nombres como: Hymes, Austin, Canale y Swain, Brumfit, Littlewood y Wilkins, entre otros. También al enunciar este enfoque, rápidamente se contrasta con enfoques 'lingüísticos', 'gramaticales', 'estructurales' y 'formales' para la enseñanza de lenguas. La mayor diferencia, se puede observar en el hecho de que las teorías formales o estructurales, conciben la lengua fuera de un contexto particular de uso, mientras que la teoría comunicativa presenta la lengua en un contexto de enunciación social y cultural específico (Stern, 1983).

El Enfoque Comunicativo, se define básicamente en relación con su objeto de estudio -la Competencia Comunicativa-. Si el enfoque estructural se fundamentaba en la idea de estudiar la lengua como un sistema compuesto de subsistemas -fonológico, sintáctico y semántico- y cómo estos operan; el Enfoque Comunicativo se enfoca en la interacción y en la relación con el contexto social. Esto es lo que Hymes (1972) llamó 'Competencia Comunicativa'. Hymes (1972) la definió como la habilidad para saber "cuándo hablar, cuándo no, y sobre qué hablar con quién, cuándo, dónde y de qué forma hacerlo” (p.277)

Es importante aclarar que las diferencias mencionadas anteriormente, comenzaron con Chomsky cuando propuso las distinciones entre Competencia y Actuación. Entendida la Competencia como la representación mental de las reglas lingüísticas, las cuales componen la gramática internalizada del hablante/receptor. Por otro lado, la Actuación consiste en la comprensión y producción de la lengua. El punto más importante es que solo mediante la Actuación se puede confirmar la existencia de la Competencia, porque las reglas que el aprendiente ha internalizado no son directamente observables. En este sentido, es necesario examinarlas solo cuando el aprendiz las produce. Esto quiere decir que sobre todo en la producción "los enunciados que el aprendiente produce son tratados como ventanas a través de las cuales puede observarse el sistema internalizado de reglas" (Ellis, 1985, p. 6). 
De hecho, la mayor diferencia entre la posición Chomskiana en relación con la Competencia y la idea de Hymes, de acuerdo con Duranti (citado en Kiesling \& Bratt, 2005), es que la primera se fundamenta en la suposición de que el conocimiento puede ser estudiado de manera separada de la actuación —concebida como la implementación del conocimiento en el uso de la lengua-, mientras que para Hymes, la participación, la actuación y el conocimiento intersubjetivo son características esenciales de la habilidad para conocer una lengua.

Sin duda alguna, la competencia comunicativa implica la competencia lingüística, pero su foco principal es la comprensión intuitiva de las reglas sociales y culturales y los significados que son expresados en cualquier enunciación (Stern, 1983). A la luz de este planteamiento, es claro que la enseñanza de lenguas tiene que reconocer las dimensiones social, interpersonal y cultural de las mismas. Sin embargo, se hace un llamado en relación a la atención sobre el hecho de cómo un aprendiz de lengua extranjera es capaz de aprender esta 'competencia comunicativa' que ni siquiera un hablante nativo reconoce y es consciente de ella.

Por ahora, solamente se señalará que cuando las personas interactúan en una lengua, que es extranjera para uno de ellos por lo menos, los significados y valores compartidos que la lengua porta no pueden obviarse fácilmente. De acuerdo con Byram \& Fleming (1998), "aprender una lengua como la habla un grupo en particular es aprender los significados, valores y prácticas compartidas de ese grupo, y que se manifiestan en esa la lengua" (p.2). Es claro que hay una consciencia de la existencia de estos significados compartidos cuando en una situación de encuentro intercultural se identifican algunos vacíos en la comunicación. Evidentemente, no es suficiente preparar a los aprendices en la lengua, sino que también es imperativo reflexionar sobre la cultura que está embebida en esa lengua.

Gracias al Enfoque Comunicativo en la enseñanza de lenguas, se puede ver que los usuarios/aprendientes de la lengua traen sus competencias generales y sus competencias específicas -las competencias comunicativaspara satisfacer sus intenciones comunicativas. La figura 1 muestra las competencias presentes en la enseñanza y aprendizaje de lenguas de acuerdo con el Marco Común Europeo de Referencia.
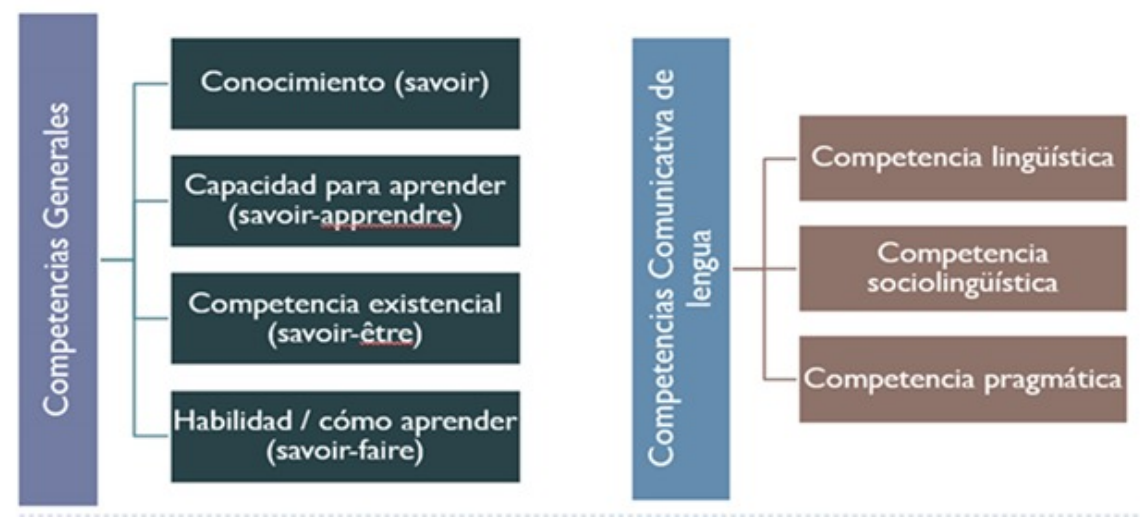

FIGURA 1

Competencias presentes en la enseñanza y aprendizaje de lenguas Fuente: Marco Común Europeo de Referencia

\section{El enfoque intercultural para la enseñanza de lenguas}

A partir de la presentación de las competencias visualizadas en la figura anterior, la pregunta que se trae a discusión es si es necesario que 'se enseñen' todas estas competencias de manera explícita o si se deben proponer situaciones a los estudiantes para que las desarrollen. Desde la perspectiva que atañe este artículo, no es suficiente ser consciente de la existencia de estas competencias; es necesario saber cómo usarlas en 'contextos 
específicos'. En otras palabras, se impone un reto mayor a los docentes de lengua en relación con la formación integral de los aprendientes.

El asumir este nuevo reto no implica cambiar enteramente las prácticas pedagógicas asumidas en los procesos de enseñanza de una lengua extranjera. Por el contrario, pone en un nuevo escenario que sugiere la no 'des-etnización' y la 'des-culturización' de las lenguas. Las lenguas son parte de la cultura y como tal, deben ser estudiadas y analizadas. La perspectiva obtenida en este estudio apunta a que el mayor problema que tiene un profesor de lengua, es creer que la lengua es sinónimo de un país o nación. De hecho, son los libros para la enseñanza de lenguas los que perpetúan esta creencia y son los docentes quienes tienen la responsabilidad de ayudar en la construcción identitaria y cultural de nuestros estudiantes. En este orden de ideas, la enseñanza de una lengua extranjera debería invitar a los aprendientes a tomar un rol de etnógrafos comparativos. Esto significa que deberían observar el mundo de manera crítica para comparar y crear sus propias hipótesis y compartir sus experiencias con los otros.

Ahora bien, esta perspectiva le exige a la enseñanza comunicativa de la lengua un reto de superar esa 'vieja' idea de 'entrenar' a los aprendientes al uso de la lengua como hablantes nativos. El hablante nativo término problemático en las sociedades plurilingües y multiétnicas actuales - no debe ser considerado como el 'modelo' de usuario de la lengua. En este sentido no se debería aceptar que 'un modelo' de comunicación, sea tipificado por el uso de la lengua que hacen los "supuestos hablantes nativos", ni mucho menos aceptar que se evalúe la competencia de un hablante no nativo a la luz de la competencia del nativo - este punto es bastante crítico para el dominio de la evaluación, que no entraré a discutir por ahora-. Finalmente, no se puede seguir pensando que para la enseñanza comunicativa el 'hablar casi como un nativo' trae a sus hablantes cierta autoridad asociada con la autenticidad y su legitimidad en el uso de la lengua (Byram \& Fleming, 1998). Por el contrario, como lo sostiene Kramsch (citada en Byram \& Fleming, 1998) esta posición debe ser reevaluada y, de hecho, eliminada. Lo que esta autora propone es un 'hablante intercultural', quien es capaz de establecer relaciones entre su propia cultura y la otra; quien puede mediar y explicar la diferencia; y quien muestra empatía con el otro. En este punto quiero entrar a discutir la importancia de incluir la Competencia Comunicativa Intercultural en la enseñanza de lenguas.

\section{El hablante intercultural y la enseñanza de lenguas}

Se ha mencionado, parcialmente, que no se puede continuar pensando que el mejor aprendiz de lenguas, es aquel que se acerca en gran medida al dominio de la gramática y el vocabulario que posee un hablante nativo. El objetivo de la enseñanza de lenguas, ya no debe ser definido solamente en términos de la adquisición de la competencia comunicativa en una lengua extranjera: los docentes ahora tienen la responsabilidad de desarrollar la Competencia Comunicativa Intercultural (CCI).

El enfrentarse a la CCI exige unas características especiales. De acuerdo con Sercu et al. (2005)

"estas características y competencias han sido identificadas como el deseo de vincularse en la cultura extranjera, la consciencia de sí mismo, y la habilidad de mirarse desde el exterior; la habilidad de actuar como un mediador cultural; la habilidad para evaluar los puntos de vista de los otros; la habilidad de emplear conscientemente las habilidades culturales de aprendizaje y de leer el contexto cultural; y el entender el hecho de que los individuos no pueden ser reducidos a sus identidades colectivas" ( $p$. 2)

En el contexto de enseñanza de lenguas extranjeras, la competencia intercultural está relacionada con la competencia comunicativa. Es decir, la habilidad para actuar apropiadamente en situaciones comunicativas donde se despliega el conocimiento lingüístico, sociolingüístico y pragmático para establecer relaciones intersubjetivas. De esta manera, la competencia intercultural, se basa en la competencia comunicativa y la expande en su comprensión. Esta competencia se caracteriza por la presencia de unos saberes (savoirs) 
los cuales están integrados y entrelazados con algunos otros componentes de la competencia comunicativa (conocimiento, habilidades y actitudes). Entre los saberes tenemos: (Sercu et al., 2005)

- El primer saber (saberes - savoirs) con la 's' del plural, constituye la dimensión del conocimiento. Este ha sido definido por Byram (citado en Sercu et al., 2005), "por un lado, como el conocimiento acerca de los grupos sociales y sus culturas y por el otro, un conocimiento de las maneras como suceden las interacciones en los niveles social e individual" (p. 4). Estos savoirs representan el rango de referencia de las personas que hacen parte de una cultura particular: las palabras y los gestos que usan las personas, sus comportamientos, sus valores, sus símbolos, entre otros, están siempre ligados a la cultura y tienen un significado dentro de esa comunidad en particular. Además del conocimiento específico de una cultura, las personas también necesitan adquirir una cierta cantidad de conocimiento de cultura general que les permita enfrentarse con una amplia diversidad de culturas extranjeras - la distinción entre la 'C' mayúscula y la 'c' minúscula-.

- El segundo saber tiene que ver con el saber aprender (savoir-apprendre) y el saber comprender (savoircomprendre). Estos constituyen las habilidades necesarias para interactuar con los otros. 'El saber aprender', según Byram y Zárate (citados en Sercu et al., 2005), se refiere a "la capacidad para aprender culturas y asignar significados a los fenómenos culturales de manera independiente” (p. 4) y 'el saber comprender' se refiere a la capacidad para interpretar y relacionar culturas. Estas habilidades además del conocimiento y las actitudes son puntos clave para el continuo aprendizaje.

- El tercer saber tiene que ver con el saber hacer (savoir-faire). Este se refiere a la habilidad general para actuar de una manera interculturalmente competente en situaciones de contacto intercultural; para tener en cuenta la identidad cultural específica del interlocutor y; para actuar de manera respetuosa y cooperativa. (Sercu et al., 2005).

- El cuarto saber corresponde al saber ser (savoir-être), que se refiere a la capacidad junto con el deseo de abandonar las actitudes y percepciones etnocentristas así como la habilidad para establecer y mantener una relación entre la cultura propia al igual que la extranjera.

- El quinto saber es el saber vincularse o hacer parte de (savoir-s'engager), que se caracteriza por un compromiso crítico con la cultura extranjera y la propia. Aquí se pone en práctica la habilidad para evaluar críticamente ambas culturas.

Lo que estos saberes sugieren, es que necesitamos ser conscientes de nuestra cultura y la de los demás con el fin de construir un puente de mutuos entendimientos interculturales. En este sentido, lo que los docentes y estudiantes necesitan, de acuerdo con Byram \& Fleming (1998), es "desarrollar una actitud positiva para aprender, comprender y apreciar la cultura de los otros sin perder su propio estatus, rol e identidad cultural" (p.116).

Es claro que una de las contribuciones que la enseñanza de lenguas extranjeras podría hacer para el desarrollo de los anteriores saberes es ayudar a los aprendientes a entender la otredad. Ya Sartre había utilizado este término para estudiar a fondo la existencia de uno mismo, a través de lo que sería la mirada del otro. En este sentido se considera que los salones para la clase de enseñanza de lengua extranjera, se deben convertir en escenarios potentes para analizar la otredad, en tanto que los aprendientes se ven confrontados con su propia lengua, con su propia cultura y con la lengua y la cultura del otro. En este orden de ideas, Byram (1989) menciona que hoy en día existen muchas situaciones en donde las personas aunque nacen en el mismo país, son percibidas como étnicamente extranjeras. Así, la 'otredad' es una característica de cualquier sociedad actual (Byram, 1989).

El planteamiento anterior, lleva a pensar si existe una preparación para enfrentarse con la 'diferencia', o si se ha recibido 'entrenamiento suficiente' para lidiar con ésta. Así las cosas, se llega a la misma conclusión que establecida anteriormente, la enseñanza de lenguas debería enfocarse no solo en el desarrollo de la competencia comunicativa, sino también de las competencias interculturales. Como Savicki (2008) afirma 
"un enfoque intercultural debería tener como objetivo preparar a los estudiantes no solo para que triunfen en el país en el que estudian sino que también para que estén preparados para triunfar en cualquier cultura" (p.11).

A esta altura de la discusión, es de vital importancia que haya el concepto de 'hablante intercultural' como aquel que es capaz de alejarse de los juicios ya construidos de su propia cultura y la explora a través de los ojos del otro. Esto contribuye al desarrollo de la consciencia crítica de sí mismo, y permite que sea observada la perspectiva del otro. Adicionalmente, "el hablante intercultural aprende que no puede asumir que los fenómenos de su propia cultura son comprendidos de la misma manera por los demás” (Tarasheva \& Davcheva, 2001, p. 47)

La enseñanza de lenguas debería comenzar a tomar seriamente el concepto de una lengua como un medio de comunicación e interacción con personas de otras culturas. Se sabe que en el aprendizaje de otra lengua, los estudiantes se exponen a uno o más grupos sociales y a sus prácticas culturales - y de manera inevitable aprenden algo de estas culturas (Byram \& Fleming, 1998). Por lo tanto no debería ser subestimado cualquier esfuerzo hecho con el fin de desarrollar la competencia comunicativa intercultural.

Evidentemente a la luz de todos estos planteamientos es necesario tener claridad sobre lo que se pone como reto en los contextos de enseñanza de una lengua extranjera. Así las cosas, surge la pregunta por el tipo de enfoque privilegiado en la enseñanza de la lengua: un enfoque de culturas extranjeras, un enfoque intercultural, un enfoque multicultural o un enfoque transcultural (Risager, 1998). A continuación, se pueden ver las diferencias entre estos enfoques.

- El enfoque de culturas extranjeras: Está basado en el concepto de una sola cultura, asociada con personas específicas, una lengua específica y normalmente, con un territorio específico. Este enfoque se centra en la cultura del país o países donde se habla la lengua (países objetivo). También ha sido llamado "el enfoque monocultural". Este no se enfoca en el país del aprendiente y tampoco en las relaciones entre los países objetivos y el del aprendiente o cualquier otro.

Este enfoque ha sido el paradigma dominante en la enseñanza de lenguas extranjeras en los países occidentales desde el siglo pasado. Hoy en día se ha puesto en duda sobre todo, porque se basa en un concepto de una cultura dominante (Risager, 1998).

- El enfoque intercultural: Se basa en el concepto de cultura que tiene como punto de partida el hecho de que diferentes culturas están estructuralmente relacionas entre ellas. Por lo tanto, este concepto abarca el encuentro de culturas, incluyendo intentos para lidiar, entender y reconocer las diferencias entre ellas.

Desde este enfoque, la enseñanza puede ser caracterizada por actitudes de relativismo cultural y por el deseo de asumir una perspectiva no etnocentrista de las culturas involucradas. Aquí la lengua objetivo es enseñada como si fuera la lengua materna para los estudiantes; sin embargo, "el objetivo es desarrollar una competencia intercultural y comunicativa, una competencia que permita al aprendiz funcionar como mediador entre dos culturas" (Risager, 1998, pp. 244-245).

Por su parte, Kramsch (1998) estipula que el término intercultural usualmente se refiere al encuentro de dos culturas o dos lenguas cruzando límites políticos de naciones o Estados. Este término también se refiere a la comunicación entre personas de distintas culturas étnicas, sociales y de género dentro de los límites de la misma lengua nacional, es así como la comunicación intercultural está asociada con asuntos de bilingüismo y biculturalismo (Kramsch, 1998).

- El enfoque multicultural: Este enfoque se basa en el hecho de que numerosas culturas pueden coexistir dentro de los límites de una de estas y dentro de la misma sociedad o Estado. Para algunos grupos en la sociedad en cuestión la lengua 'nacional' o estándar/oficial es su lengua materna; para otros es su segunda lengua; para otros es una lengua extranjera. Este enfoque se centra en la diversidad étnica y lingüística del país o países objetivo. También se enfoca en la diversidad étnica y lingüística del país de los aprendientes y las relaciones migratorias de ambos. (Risager, 1998) 
Kramsch (1998) afirma que el término 'multicultural' se emplea frecuentemente de dos formas. En un sentido social, éste indica la coexistencia de personas de numerosos orígenes y etnias como en las 'sociedades multiculturales'. En un sentido individual, con este término se caracteriza a las personas que pertenecen a varias comunidades discursivas y quienes, por lo tanto, tienen los recursos lingüísticos y las estrategias sociales para afiliarse e identificarse con varias culturas y con varias formas para emplear la lengua (Kramsch, 1998).

- El enfoque transcultural: Este enfoque tiene como punto de partida el carácter entrelazado de las culturas como una condición común para todo el mundo: las culturas se penetran en combinaciones cambiantes dependiendo de la migración y el turismo, los sistemas de comunicación global para las masas y la comunicación privada, la interdependencia económica, y la globalización de la producción de bienes. El uso extensivo de un número de lenguas como lingua francas es una de las expresiones de un mundo internacionalizado.

El enfoque transcultural se centra en la vida de individuos y grupos en contextos caracterizados por su, más o menos, complejidad cultural y lingüística: los canales de televisión, la calle en la ciudad, el salón multicultural, las situaciones de supermercado en donde la lengua meta es empleada pero, de tal forma que los aprendientes también son conscientes de que otras lenguas son empleadas.

Ya para finalizar y con el fin de dar orden a la plétora de conceptos relacionados con la competencia comunicativa intercultural, se propone realizar una distinción inicial entre comunicación, lengua y cultura, y ver sus relaciones junto con sus conexiones con el aprendizaje intercultural.

\section{Comunicación, lengua y cultura}

\section{¿Qué es comunicación?}

Se ha dicho que la comunicación no es simplemente el intercambio de información o un medio a través del cual las personas envían información. Como Rico (2004) afirma, la comunicación es un proceso intersubjetivo por el cual las personas negocian significados. No es un proceso predecible donde se pueda inferir qué va a suceder; por el contrario, es un proceso en el que los individuos constantemente construyen, significan y resignifican significados. Thompson (2003) asevera que "la comunicación tiene un rol mucho más importante y extenso de lo que muchas personas generalmente piensan, es un proceso a través del cual la identidad de un individuo se constituye" (p. 26).

Fiske afirma que "la comunicación es una de esas actividades humanas que todos reconocen pero pocos pueden definir satisfactoriamente" (citado en Thompson, 2003, p. 9). Esto ocurre porque la comunicación es una pieza perfectamente integrada en el diario vivir que se tiene a dar por sentada, raramente al detenerse para considerar lo que involucra o cuán importante es para nosotros.

Adicionalmente, Fiske brinda, la versión más corta y concreta del significado de comunicación: la comunicación "son interacciones sociales a través de mensajes" (Fiske citado en Thompson, 2003, p. 10). Se puede ver que esta definición está compuesta por tres conceptos clave: 'interacción, social y mensaje'. El primer concepto de esta definición es 'interacción'. Indudablemente, la comunicación involucra interacción, y esto se entiende como "el intercambio colaborativo de pensamientos, sentimientos o ideas entre dos o más personas, que tiene como resultado un efecto recíproco en cada uno" (Brown, 2001, p.164). Quisiera adicionar a esta definición que la interacción siempre está mediada. Esta mediación se logra gracias a unas herramientas claves: el lenguaje verbal y no verbal, el paralenguaje, y el uso de otros canales como los textos impresos o visuales y el uso de la tecnología.

El segundo concepto es el 'social'. Thompson (2003) asevera que la comunicación es un evento social que tiene una dimensión política e ideológica social y que ocurre en un contexto y éste a su vez la moldea. Es 
claro que la comunicación no ocurre en el vacío, siempre se adscribe a un contexto y a unos interlocutores que portan unos intereses específicos. Finalmente, el tercer término es el 'mensaje'. Cuando se comunica, se estan negociando significados mediante actos de habla. Estos son intenciones de los hablantes y son formalizados así como representados mediante mensajes. De esta manera, lo que finalmente se comunica son solo mensajes. Frente a esta postura, Thompson (2003) dice que

deberíamos ser precavidos de asumir que la comunicación siempre es intencional. Un mensaje puede ser enviado y recibido incluso si la persona que se comunica no tiene intensión de hacerlo. Por ejemplo, si mi tono de voz y mi comunicación no verbal indican nerviosismo, es muy probable que esté comunicando ese nerviosismo, aunque realmente no desee hacerlo (p.11).

Lo que Thompson (2003) declara es precisamente lo que hace a la comunicación un fenómeno social y humano mucho más interesante. Lo que se comunica por un medio es diferente a lo que se comunica por otro. La idea es hacerse consciente de esos medios e intentar expresar y negociar significados; es por esta razón que la comunicación es un evento que se enmarca en la esfera 'intersubjetiva'. Algunas veces se logran acuerdos, otras veces no; en algunos momentos se comprende, en otros se llega a malinterpretar: la comunicación no es predecible. En conclusión, un punto importante que debe ser reconocido sobre la comunicación es que no es una pieza básica de en la vida diaria, sino que es una parte fundamental. En este sentido, Thompson (2003) estipula que "no podemos no comunicarnos" (p.11).

En cuanto a la lengua, es interesante reflexionar sobre la idea planteada por Holliday, Hyde \& Kullman (2004) cuando dice: "hablas por lo tanto existes" (p.184)

\section{¿Qué es lengua?}

Gracias a la lengua es posible expresar la manera cómo se piensa, la forma en la de interactuar con el otro, la manera como en que se ama, cuando se odia y; en términos generales, la lengua permite estar en el mundo. Durante muchos años, la enseñanza de lenguas ha tomado a la lengua como el medio por el cual las personas se comunican. En otras palabras, ésta se veía como una herramienta de comunicación, "un método que los humanos usan para indicar objetos e ideas de su mundo físico y social” (Bennet, 1998, p.12). Como se mencionó al principio de este artículo, la lengua se concibe como un sistema que tiene que ser aprendido por partes - la perspectiva estructuralista-. Hasta hace poco, la lengua ha recibido la importancia que merece. Byram (2006) establece que "las lenguas simbolizan identidades y son empleadas para señalar identidades por quienes las hablan". (p.5)

Además, las lenguas dan un sentido de pertenencia cultural a un grupo - local, regional o nacional. "Es un conjunto de relaciones entretejidas en las que una forma lingüística acepta su significado dado su lugar dentro del total del sistema de signos" (Thompson, 2003, p.37). Esta es la base de las interacciones interpersonales y sociales en diferentes niveles, al igual que es la base del pensamiento, de los sentimientos, acciones e interacciones; "también es un factor primario en términos de la creación de la sociedad en relación con los factores culturales y estructurales" (Thompson, 2003, p. 36).

Adicionalmente, "la lengua es un puente entre personas, pero también es un muro que las divide" (Holliday et al, 2004, p.184). Tan pronto es utilizada la lengua, somos juzgados por las personas como pertenecientes a ciertos grupos sociales y, de esta forma, aparecen imágenes y estereotipos en relación con nuestra cultura. En esta línea, Byram (1989) establece que la lengua enmarca de forma relevante los valores y significados de una cultura; se refiere a artefactos culturales y; señala la identidad cultural de las personas.

En los estudios interculturales, la lengua se convierte en un fenómeno social altamente relevante. A través de la lengua las personas pueden vivir juntas o pueden estar separadas. No es necesarios ser expertos en las ciencias del lenguaje para ver la importancia de la lengua en la vida humana; con la lengua es posible crear 
mejores lugares para vivir o los peores ambientes para habitar. En esta línea, Thompson (2003) afirma que se debería evitar la lengua que se usa para:

- Excluir: Algunas formas de la lengua implican que ciertas actividades (aquellas asociadas con las posiciones de poder) están reservadas para ciertas personas.

- Despersonalizar: Se refiere al uso de la lengua que despoja a las personas de su individualidad o incluso de su humanidad.

- Estigmatizar: Algunas formas de la lengua tienen connotaciones negativas, por lo que se debe ser cuidadoso de cómo son emplean - si es que es necesario utilizarlas.

- Reforzar estereotipos: Los estereotipos discriminatorios no son solamente destructivos e inútiles, sino que también tienen un efecto distorsivo en la comunicación. Los estereotipos pueden ser perpetuados a través del lenguaje que utilizado.

- Legitimar la discriminación: El término 'legitimar' significa presentar algo como aceptable o legítimo. Algunas formas de la lengua implican que la discriminación es aceptable.

De acuerdo con Thompson (2003), la lista puede expandirse si se consideran los casos del uso de la lengua para ofender o en situaciones de humor. Las personas pueden decir fácilmente 'era solo una broma' y, mediante esto, no se considera discriminatorio u ofensivo para un grupo particular o un individuo en específico. La figura 2 deja ver algunas formas problemáticas en las que se usa la lengua.

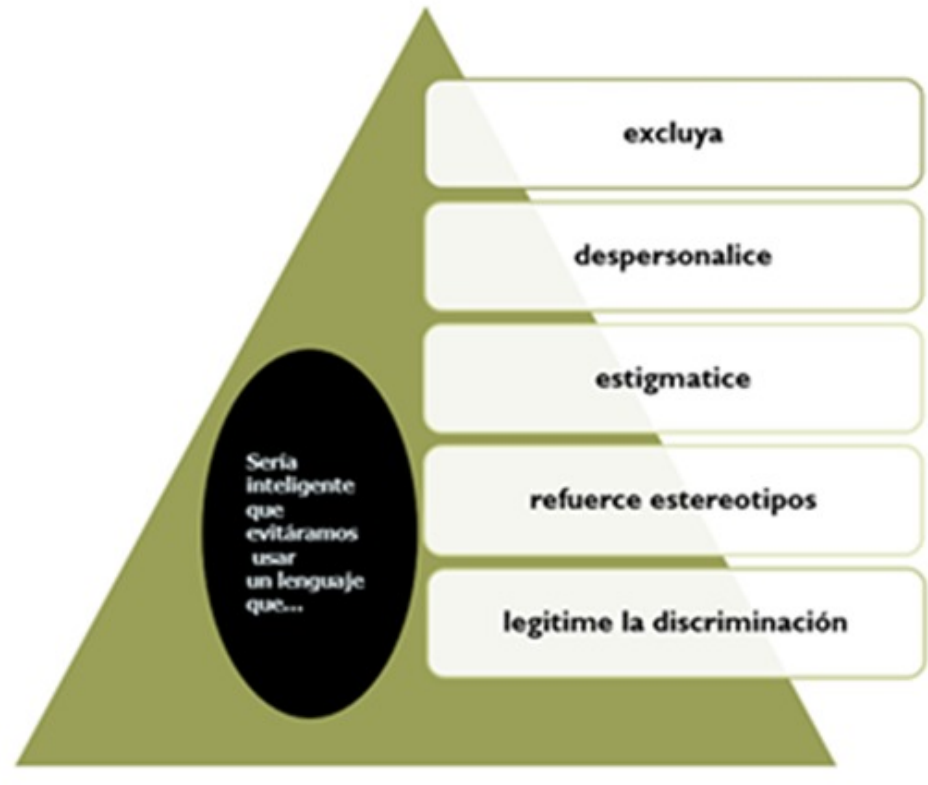

FIGURA 2

Formas problemáticas de la lengua

Fuente: Thompson, 2003, p.139

En resumen, Thompson (2003) no propone un enfoque defensivo en el uso de la lengua ni tampoco sugiere restringir sus características. Lo que se debería hacer, según sus sugerencias, es desarrollar una sensibilidad en la que se encuentre preparado para aprender de los errores, y despertar la consciencia del significado de la lengua, de tal forma que estos inconvenientes puedan ser discutidos y debatidos en profundidad. "Sin ese debate, las personas no aprenderán y, en consecuencia, continuarán empleando un lenguaje poco incluyente o tomarán una posición defensiva que no permitirá alcanzar una comunicación efectiva” (Thompson, 2003, p.140).

En relación con la cultura, se trae una analogía sobre cultura presentada por Fennes \& Hapgood (1997): "La cultura es como un pez intentando entender el agua - el agua es algo que rodea al pez todo el tiempo, haciendo 
parte de esta y sin poder escapar de ella" (p. 13). La cultura nos rodea todo el tiempo y, por lo tanto, no es posible percibirla de manera objetiva. Aunque se intente definir cultura, esta definición está condicionada por nuestro entorno - cultural. No se puede percibir la existencia de la cultura porque somos parte de ella (así como la existencia del agua para el pez); solamente se puede notar su existencia, cuando somos confrontados por algo distinto.

\section{¿Qué es cultura?}

Es claro que cuando somos confrontados por otro 'entorno' - otra cultura - es posible que no se logre entender enteramente este 'otro' entorno. Mientras que la consecuencia para el pez sea la muerte, para los seres humanos puede resultar en un 'choque cultural'. Volviendo a la analogía, Fennes \& Hapgood (1997) afirman que solo aplica en cierta medida en el sentido de que "un pez no da forma al agua en la que nada, mientras que la cultura es moldeada por quienes hacen parte de ésta” (p.14). En consecuencia, la cultura no es estática sino dinámica; está en un continuo cambio y transformación que está determinada no solo por influencias internas sino también externas de otros individuos y culturas.

Es cierto que damos forma a la cultura, pero la cultura también nos moldea. Los grupos en los que hemos crecido nos han condicionado para ver el mundo desde sus propias perspectivas; por ejemplo, ¿por qué salivamos cuando pensamos en comer carne de res? De acuerdo con Bennett (1998), depende de qué tan exhaustivo hemos internalizado las actitudes y valores que nos han enseñado nuestros grupos. Este autor afirma que "no solo las lenguas que hablamos y la manera en que pensamos, sino que incluso lo que vemos, escuchamos, saboreamos, tocamos y olemos están condicionados por las culturas en las que hemos crecido" (Bennett, 1998, p. 98).

Siguiendo con las analogías, Fennes \& Hapgood (1997) compararon la cultura con un iceberg en cuanto que solo un pequeño porcentaje es visible (Figura 3) La parte más grande de lo que compone la cultura está más allá de nuestra percepción; esto no significa que tenga menor influencia en nuestras vidas. Sabemos cómo actuar y comportarnos de acuerdo con la cultura, pero no somos conscientes de ella y, en consecuencia, no podemos controlarla. Lo que para algunos puede ser obvio para otros no lo será. 


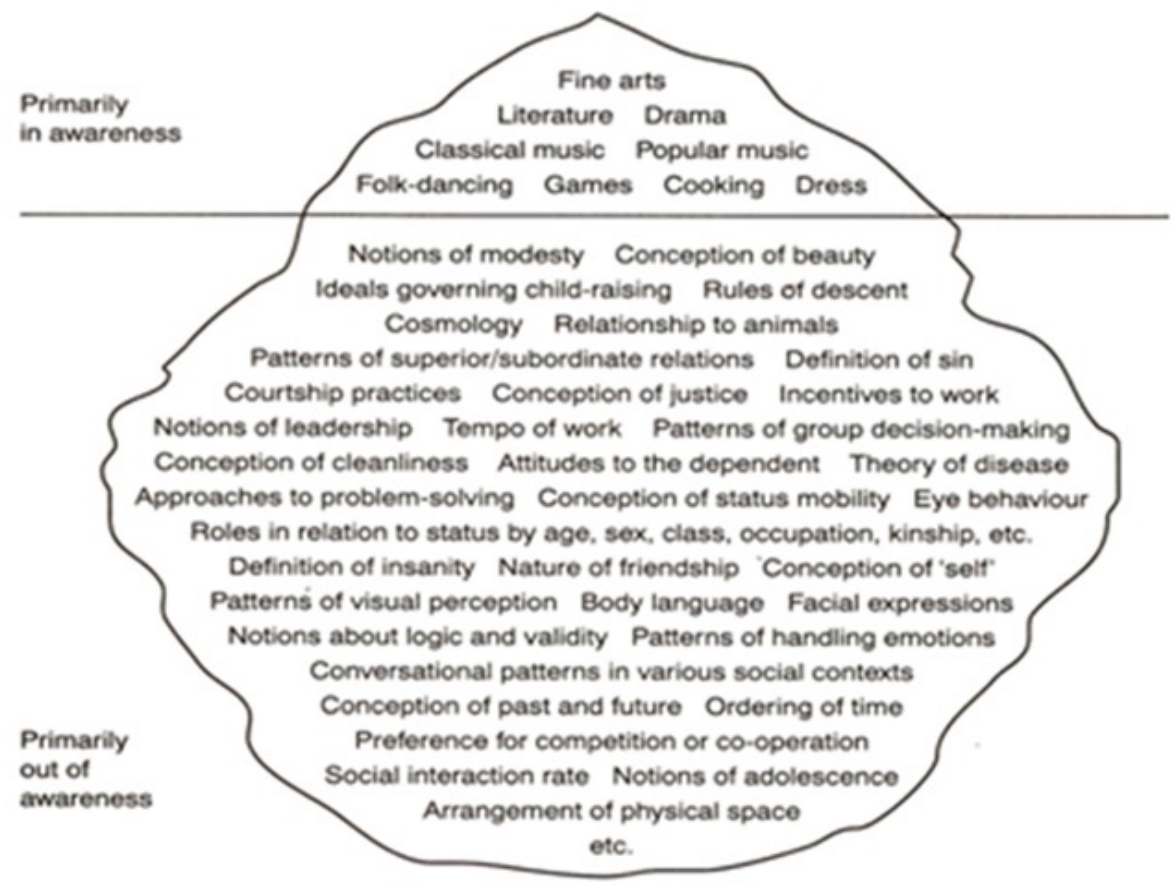

FIGURA 3

El concepto del iceberg de la cultura

Fuente: Fennes \& Hapgood, 1997, p.14

Inferimos de la analogía del iceberg que existen dos perspectivas a través de las cuales se ha comprendido la cultura. Bennett (1998) las distingue como "Cultura en mayúscula" y "cultura en minúscula". La primera se refiere al tipo de cosas incluidas en las áreas de estudio o en los cursos de historia ('cultura objetiva'): literatura, drama, artes, música clásica, etc. El estudio de estas áreas, constituye la mayor parte tanto del currículo internacional como el de educación multicultural. El segundo se refiere a las características psicológicas que definen un grupo de personas - su pensamiento y comportamiento cotidiano - más que a las 'instituciones' que se ha creado. Desde esta perspectiva, la cultura es "los patrones aprendidos y compartidos de creencias, comportamientos y valores de los grupos que interactúan" ('cultura subjetiva') (Bennett, 1998, p.3). La diferencia se ha llamado en términos más académicos como 'cultura objetiva' y 'cultura subjetiva' respectivamente. En conclusión, Bennet asevera que la tradicional educación internacional multicultural usualmente se enfoca en la 'cultura objetiva' (Cultura en mayúscula), mientras que la comunicación intercultural se enfoca exclusivamente en la 'cultura subjetiva (cultura en minúscula)'.

El interés de este estudio radica en que a pesar de los innumerables intentos para definir el concepto de cultura, llegar a una definición apropiada no es fácil. Se puede decir que, a partir de todas esas definiciones, la mayoría llega a la misma conclusión: la cultura es la representación simbólica de la vida cotidiana de un grupo. Por vida cotidiana se hace una referencia a los rituales de interacción, los estilos de vida y los objetos empleados por un grupo (también denominados artefactos). Entender la cultura permite la interpretación de la vida social y, de esta forma, ofrece una orientación sobre las acciones y comportamientos de un grupo; en este sentido, la cultura es vista como un proceso dinámico.

De acuerdo con Fennes \& Hapgood (1997), el Centro Contemporáneo de Estudios Culturales de la Universidad de Birmingham emplea un enfoque mucho más refinado en torno a la cultura, el cual afirma que:

La cultura de un grupo o clase incluye el tipo de vida único y especial de este grupo o clase; los significados, los valores y las ideas reflejadas en las instituciones, en las relaciones sociales, en el sistema de creencias, en las costumbres y las tradiciones, en el uso de objetos y en la vida material. La cultura es la forma específica en la que esta organización material y social se expresa. 
La cultura incluye un 'mapa de significados', el cual hace que las cosas sean comprensibles para sus miembros. Estos 'mapas de significados' no se ubican en nuestros cerebros: están representados en las formas de organización social y de relaciones a través de las cuales el individuo se convierte en un 'individuo social'. La cultura es la manera en la que las relaciones de un grupo se estructuran y moldean; sin embargo, también es la manera en que se experimentan, se entienden y se interpretan. (p.15)

Como ya se ha mencionado anteriormente, la cultura es dinámica y representa el estilo de vida de un grupo en donde, de acuerdo con Larcher (citado en Fennes \& Hapgood, 1997), las partes visibles del iceberg son las leyes, las reglas, las costumbres y las tradiciones; mientras que el comportamiento rutinario y los hábitos son inconscientes y, de esta forma, no son visibles.

Es así como, el punto de consideración es cómo hacer más visibles los aspectos invisibles de la cultura. La respuesta es menos compleja de lo que parece: solo cuando se enfrenta lo extranjero y se puede ver la diferencia, es posible notar quiénes somos. Es en ese escenario en dónde puede desarrollarse la competencia intercultural que ayuda a tratar de mejor manera la diferencia.

Ya para finalizar son generadas algunas reflexiones en torno a pensar los escenarios de enseñanza de lenguas como uno de los contextos en los cuales es posible estar tratando apropiadamente la divergencia y la diversidad en situaciones en donde como interlocutores, se asumen diferentes identidades sociales y culturales.

Indudablemente una forma posible de tratar apropiada y efectivamente la diversidad sea étnica, racial, religiosa, de género o cultural es a través del desarrollo de la Competencia Comunicativa Intercultural (CCI). Esta competencia debería estar en la agenda de cualquier programa de enseñanza de las lenguas extranjeras.

\section{Referencias}

Bennett, M. (1998). Basic concepts of intercultural communication. Selected readings. Boston USA: Intercultural Press. A. Nicholas Brealey Publishing Company.

Brown, H.D. (2001). Teaching by principles. An interactive approach to language pedagogy. New York USA: Longman. Buttjes, D. \& Byram, M. (1991). Mediating languages and cultures. Clevedon, Avon: Multilingual Matters.

Byram, M. (1989). Cultural studies in foreign language education. Clevedon UK: Multilingual Matters Ltd.

Byram, M. (2006). Languages and identities. Strasbourg: Council of Europe.

Byram, M. \& Fleming, M. (1998). Language learning in intercultural perspective. Approaches through drama and ethnography. Cambridge: Cambridge University Press.

Kiesling, S., \& Bratt, C. (Eds). (2005). Intercultural discourse and communication. The essential readings. Malden USA: Blackwell Publishing Ltd.

Ellis, R. (1985). Understanding second language acquisition. Oxford: Oxford University Press.

Fennes, H. \& Hapgood, K. (1997). Intercultural learning in the classroom. Crossing borders. London UK: Cassel Council of Europe Series.

Holliday, A., Hyde, M., \& Kullman, J. (2004). Intercultural communication. An advance resource book. London UK: Routledge Applied Linguistics.

Hymes, D. (1972). On communicative competence. En J.P. Holmes (Eds.), Sociolinguistics (pp. 269-293). Harmondsworth: Penguin.

Kramsch, C. (1993a). Language study as border study: experiencing difference. European Journal of Education, 18(3), 349-358. https://doi.org/10.2307/1503764

Kramsch. C. (1993b). Context and Culture in Language Teaching. New York: Oxford University Press

Richards, J.C. (1986). Approaches and methods in language teaching. A description and analysis. Cambridge: Cambridge University Press.

Rico Troncoso, C. (2004). Reflexiones del quehacer evaluativo del ICFES en los últimos años: análisis y propuestas para mejorar el sistema educativo, ICFES, Evaluación por competencias: lenguaje e idioma extranjero (pp. 140-165). Bogotá: ICFES y Editorial Magisterio. 
Rico Troncoso, C. (2005). Searching for coherence in language teaching: the issue of teaching competences. Colombian Applied Linguistics Journal, (7), 95-107. Recuperado de http://www.scielo.org.co/scielo.php?script=sci_arttext \&pid=S0123-46412005000100007\&lng=en\&tlng=en

Rico Troncoso, C. (2010). The effects of language material son the development of intercultural competence. En B. Tomlinson \& H. Masuhara (Eds.), Research for materials development in language learning (pp. 83-102). London: Continuum.

Risager, K. (1998). Language teaching and the process of European integration. En M. Byram \& M. Fleming (Eds.), Language learning in intercultural perspective: approaches through drama and ethnography (pp. 242-254). Cambridge: Cambridge University Press.

Savicki, V. (2008). Developing intercultural competence and transformation. Theory, research and application in international education. Virginia, United States of America: Stylus Publishing.

Sercu, L., Bandura, E., Castro, P., Davcheva, L., Laskaridou, C., ... Ryan, P. (2005). Foreign language teachers and intercultural competence: An international investigation. Clevedon UK: Multilingual Matters.

Stern, H. (1983). Fundamental Concepts of Language Teaching. Oxford: Oxford University Press

Tarasheva, E. \& Davcheva, L. (2001). Up the hill of identity. En M. Byram, A. Nichols \& D. Stevens (Eds.), Developing intercultural competence in practice (pp. 44-59). Clevedon UK: Multilingual Matters.

Thompson, N. (2003). Communication and language. Hampshire: Palgrave MacMillan.

\section{Notas}

* Artículo de reflexión.

Licencia Creative Commons CC BY 4.0

Cómo citar: Rico, C. (2018). La competencia comunicativa intercultural (CCI) en los contextos de enseñanza del inglés como lengua extranjera. Signo y Pensamiento, 37(72), 77-94. https:// doi.org/10.11144/Javeriana .syp37-72.ccic 